33. Wang J, Tanderup K, Cunha A et al (2017) Magnetic resonance imaging basics for the prostate brachytherapist. Brachytherapy 16:715-727. https://doi.org/10.1016/j.brachy.2017.03.004

34. Woodrum DA, Kawashima A, Karnes RJ et al (2013) Magnetic resonance imaging-guided cryoablation of recurrent prostate cancer after radical prostatectomy: initial single institution experience. Urology 82:870-875. https://doi.org/ 10.1016/j.urology.2013.06.011

35. Woźnicki P, Westhoff N, Huber T et al (2020) Multiparametric MRI for prostate cancer characterization: combined use of radiomics model with PIRADS and clinical parameters. Cancers. https://doi. org/10.3390/cancers12071767

36. Yang F, Ford JC, Dogan N et al (2018) Magnetic resonance imaging (MRI)-based radiomics for prostate cancer radiotherapy. Transl Androl Urol 7:445-458. https://doi.org/10.21037/tau.2018.06. 05

37. Leitlinienprogramm Onkologie (Deutsche Krebsgesellschaft, Deutsche Krebshilfe, AWMF) (2019) Interdisziplinäre Leitlinie der Qualität S3 zur Früherkennung, Diagnose und Therapie der verschiedenen Stadien des Prostatakarzinoms, Langversion 5.1 (AWMFRegisternummer:043/022OL)
Radiologe $2021 \cdot 61: 838$

https://doi.org/10.1007/s00117-021-00897-1

Online publiziert: 26. Juli 2021

c Springer Medizin Verlag GmbH, ein Teil von Springer Nature 2021

\title{
Erratum zu: Neurochirurgische Eingriffe bei Traumata der Halswirbelsäule
}

\section{Stefan Linsler · B. Reyes Medina}

Klinik für Neurochirurgie, Universitätsklinikum des Saarlandes, Homburg/Saar, Deutschland

\section{Erratum zu:}

\section{Radiologe 2021}

https://doi.org/10.1007/s00117-021-008

81-9

In dem ursprünglich erschienenen Artikel war leider der deutsche Beitragstitel fehlerhaft wiedergegeben. Dieser wurde korrigiert.

Wir bitten um Beachtung.

Die Redaktion

\section{Korrespondenzadresse}

PD Dr. med. Stefan Linsler, MHBA

Klinik für Neurochirurgie, Universitätsklinikum des Saarlandes

Kirrberger Str. Gebäude 90.5, 66421 Homburg/

Saar, Deutschland

Stefan.linsler@uks.eu
Die Online-Version des Originalartikels ist unter https://doi.org/10.1007/s00117-021-00881-9 zu finden. 